JOURNAL OF

APPLIED

CRYSTALLOGRAPHY

ISSN 1600-5767

Received 5 May 2018

Accepted 8 August 2018

Edited by S. Sasaki, Tokyo Institute of

Technology, Yokohama, Japan

Keywords: piezoelectricity; ferroelectricity; in situ X-ray diffraction.
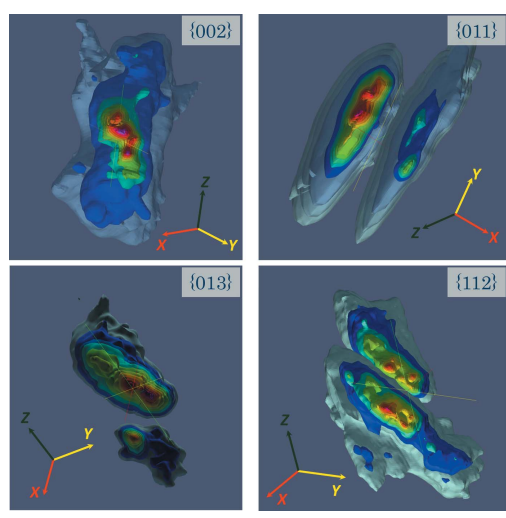

C 2018 International Union of Crystallography

\section{Probing the intrinsic and extrinsic origins of piezoelectricity in lead zirconate titanate single crystals}

\author{
Nan Zhang, ${ }^{\mathrm{a}, \mathrm{b}}$ Semën Gorfman, ${ }^{\mathrm{c} *}$ Hyeokmin Choe, ${ }^{\mathrm{d}}$ Tikhon Vergentev, $^{\mathrm{e}}$ Vadim \\ Dyadkin, ${ }^{\mathrm{f}}$ Hiroko Yokota, ${ }^{\mathrm{g}}$ Dmitry Chernyshov, ${ }^{\mathrm{f}, \mathrm{e}}$ Bixia Wang, ${ }^{\mathrm{b}}$ Anthony Michael \\ Glazer, ${ }^{\text {h }}$ Wei Ren ${ }^{\mathrm{a}}$ and Zuo-Guang Ye ${ }^{\mathrm{b}}$
}

\begin{abstract}
${ }^{a}$ Electronic Materials Research Laboratory, Key Laboratory of the Ministry of Education and International Center for Dielectric Research, School of Electronic and Information Engineering, Xi'an Jiaotong University, Xi'an, People's Republic of China, ${ }^{\mathbf{b}}$ Department of Chemistry and 4D LABS, Simon Fraser University, Burnaby, Canada V5A 1S6, ${ }^{\mathbf{c}}$ Department of Materials Science and Engineering, Tel Aviv University, Wolfson Building for Mechanical Engineering, Tel Aviv, 6997801, Israel, 'dDepartment of Physics, University of Siegen, Walter-Flex Strasse 3, Siegen, 57072, Germany, ${ }^{\mathbf{e}}$ Peter the Great St Petersburg Polytechnic University, St Peterburg, Russian Federation, 'BM01A, SNBL at ESRF, BP 220, Grenoble, 38000, France, ${ }^{\mathbf{g}}$ Department of Physics, University of Chiba, 1-33 Yayoi-cho, Inage-ku, Chiba, Japan, and ${ }^{\mathbf{h}}$ Physics Department, University of Oxford, Clarendon Laboratory Parks Road, Oxford OX1 3PU, UK. *Correspondence e-mail: gorfman@tauex.tau.ac.il
\end{abstract}

The physical origin of the piezoelectric effect has been the focus of much research work. While it is commonly accepted that the origins of piezoelectricity may be intrinsic (related to the change of lattice parameters) and extrinsic (related to the movement of domain walls), their separation is often a challenging experimental task. Here in situ high-resolution synchrotron X-ray diffraction has been combined with a new data analysis technique to characterize the change of the lattice parameters and domain microstructure of a $\mathrm{PbZr}_{1-x} \mathrm{Ti}_{x} \mathrm{O}_{3}(x=0.45)$ crystal under an external electric field. It is shown how 'effective piezoelectric coefficients' evolve upon the transition from purely 'intrinsic' effects to 'extrinsic' ones due to domain-wall motion. This technique and corresponding data analysis can be applied to broader classes of materials and provide important insights into the microscopic origin of their physical properties.

\section{Introduction}

The piezoelectric effect describes the mechanical response of a solid to an external electric field. It is implemented in such devices as pressure sensors, actuators, car-fuel injectors and frequency generators (Haertling, 1999; Wersing et al., 2008; Helke et al., 2008). The piezoelectric effect is especially strong in many structure derivatives of cubic perovskites with the general chemical formula $A B \mathrm{O}_{3}$ (Uchino, 2015; Mitchell, 2002). The perovskite's flexible structure offers a variety of possible distortions and phase transitions (Howard \& Stokes, 2005; Torgashev et al., 2005). Most importantly, the transition from the parent cubic to a lower-symmetry phase introduces distortive long-range-order parameters, like electric polarization and mechanical strains, thus creating ferroic domains (Devonshire, 1954). Even within a single crystal, such distortions are inhomogeneous and affected by defects and compositional fluctuations. Complex mesoscopic patterns of ferroic domains and domain walls play an important role in the enhancement of the piezoelectric effect: domain switching and domain-wall motions give rise to the so-called 'extrinsic' effects. On the other hand, unit-cell deformation gives rise to 'intrinsic' effects. The total macroscopic effect depends on the density of domains, their orientations, polarizations, the strain 
relationship within the domains and, most importantly, the ability of domain walls to move. Separating 'intrinsic' and 'extrinsic' mechanisms is still a challenging experimental task, especially in sintered polycrystalline ceramics where the adjacent crystalline grains are mechanically clamped to each other.

$\mathrm{X}$-ray and neutron diffraction offer the possibility to distinguish between intrinsic and extrinsic origins of the piezoelectric response (Kugel \& Cross, 1998; Durbin et al., 1999; Hall et al., 2004, 2008; Jones et al., 2005, 2006; Jones, 2007; Daniels et al., 2014; Oddershede et al., 2015; Zhang et al., 2018). Intrinsic contributions are those which cause angular shifts of the Bragg peaks, while the extrinsic contributions are those which cause intensity exchange between different peak components. Thus, careful observation of Bragg peak profiles under an external electric field serves as a probe of the intrinsic and extrinsic contributions.

However, the angular resolution of standard X-ray diffraction experiments is rarely sufficient to separate individual Bragg peak components, especially in the case of powder diffraction. As a result, the electric field induced response can only be observed as a global shift of the centre of mass (COM) of a broad Bragg intensity distribution. To discriminate between intrinsic and extrinsic contributions one should maximize the angular resolution of the X-ray diffraction experiment by, for example, using single crystals, where different domains diffract into separate peaks. The diffraction experiment must be performed under an external electric field
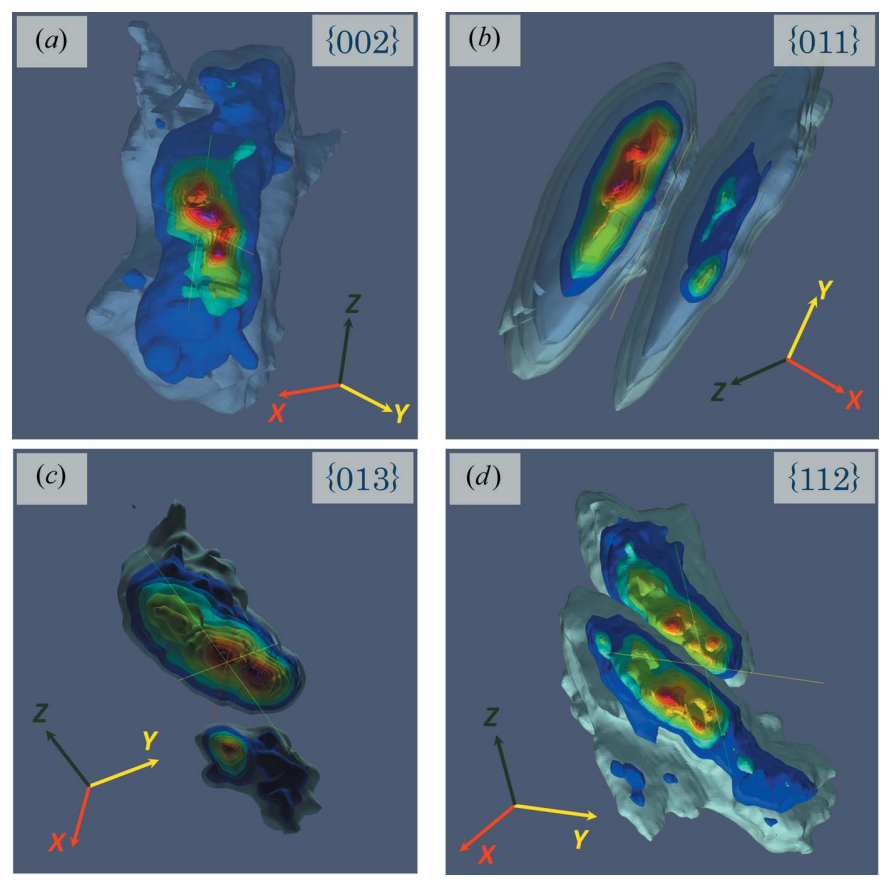

Figure 1

Three-dimensional reconstruction of the intensity distributions in the reciprocal space around four $\{h k l\}$ sets of Bragg reflections. The Cartesian coordinate axes $X Y Z$ were chosen according to the low-indexed crystallographic directions and so that the $X$ axis is parallel to the scattering vector. (a) \{002\}: $\mathbf{X}\|[0 \overline{1} 0], \mathbf{Y}\|[100], \mathbf{Z} \|[001] ; \quad(b)\{011\}$ :

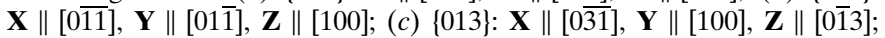
(d) $\{112\}: \mathbf{X}\|[\overline{121}], \mathbf{Y}\|[10 \overline{1}], \mathbf{Z} \|[1 \overline{1} 1]$. and synchronization must be implemented. A suitable technique has been recently introduced at the BM01 beamline of the Swiss-Norwegian Beam Lines (SNBL) at the ESRF, Grenoble (Vergentev et al., 2016; Choe et al., 2017; Vergentev et al., 2015). This is based on a diffractometer with flexible goniometry and a fast pixel area detector (Dyadkin et al., 2016), a custom-built sample environment cell (Vergentev et al., 2015), and a data acquisition scheme with a dedicated microcontroller (Choe et al., 2017).

This paper describes such a high-resolution X-ray diffraction experiment on a crystal of $\mathrm{PbZr}_{0.55} \mathrm{Ti}_{0.45} \mathrm{O}_{3}$ (PZT45) under an external electric field. High-resolution threedimensional intensity distributions were collected in reciprocal space and multiple Bragg components were separated. The shifts and exchange of intensity between individual peaks were assessed separately to reconstruct how an electric field induced strain evolves upon the inclusion of different domains in the probed material volume.

\section{Experimental}

The experiment was carried out on the PILATUS@SNBL single-crystal diffractometer (Dyadkin et al., 2016) at the European Synchrotron Radiation Facility. A PZT45 single crystal was grown using the top-seeded solution growth technique (Xie, 2013). The composition of the crystal was checked by energy-dispersive X-ray spectroscopy. The crystal was cut and polished in the form of a $0.1 \times 0.1 \times 1 \mathrm{~mm}$ needle. The longer edges of the needle were parallel to the [001] direction. ${ }^{1}$ The ends of the needle were silver-glued into a custom-built sample cell (Vergentev et al., 2016, 2015), which allows the application of an electric field in the direction parallel to the length of the needle and perpendicular to the $\mathrm{X}$-ray beam. After the determination of the orientation matrix, we measured a few prominent Bragg peaks with a resolution of $\sim 10^{-3}$ reciprocal lattice units. A cyclic electric field comparable to the coercive field was applied to the crystal, with each cycle consisting of four $(+14,0,-14$, $0 \mathrm{kV} \mathrm{cm}^{-1}$ ) consecutive electric field states, where $14 \mathrm{kV} \mathrm{cm}^{-1}$ is the maximum achievable electric field without air discharge. The duration of each state was controlled by a PILATUS $2 \mathrm{M}$ detector: the detector sent a clock signal before the acquisition of each detector frame, which was then processed by a custombuilt microcontroller to switch the electric field between two consecutive values (Choe et al., 2017).

\section{Results and discussion}

Fig. 1 displays the three-dimensional intensity distribution around four selected sets of Bragg twin peaks: $\{002\},\{011\}$, $\{013\}$ and $\{112\}$. $^{2}$ The presence of so many reflection components is due to the complex multi-domain microstructure/

\footnotetext{
${ }^{1}$ We use the pseudocubic cell setting $(a \simeq 4.1 \AA$ ) for indexing of lattice directions and Bragg reflections throughout the paper.

${ }^{2}$ The notation $\{h k l\}$ denotes the set of Bragg reflections (or Miller indices of lattice planes) that are obtained by applying 48 symmetry operations of the crystallographic point group $m \overline{3} m$ to the indices $h k l$. In cubic symmetry, the lengths of all 48 such reciprocal lattice vectors are equal. For lower symmetry, the lengths of the vectors differ, giving rise to splitting characteristic of each crystal system.
} 

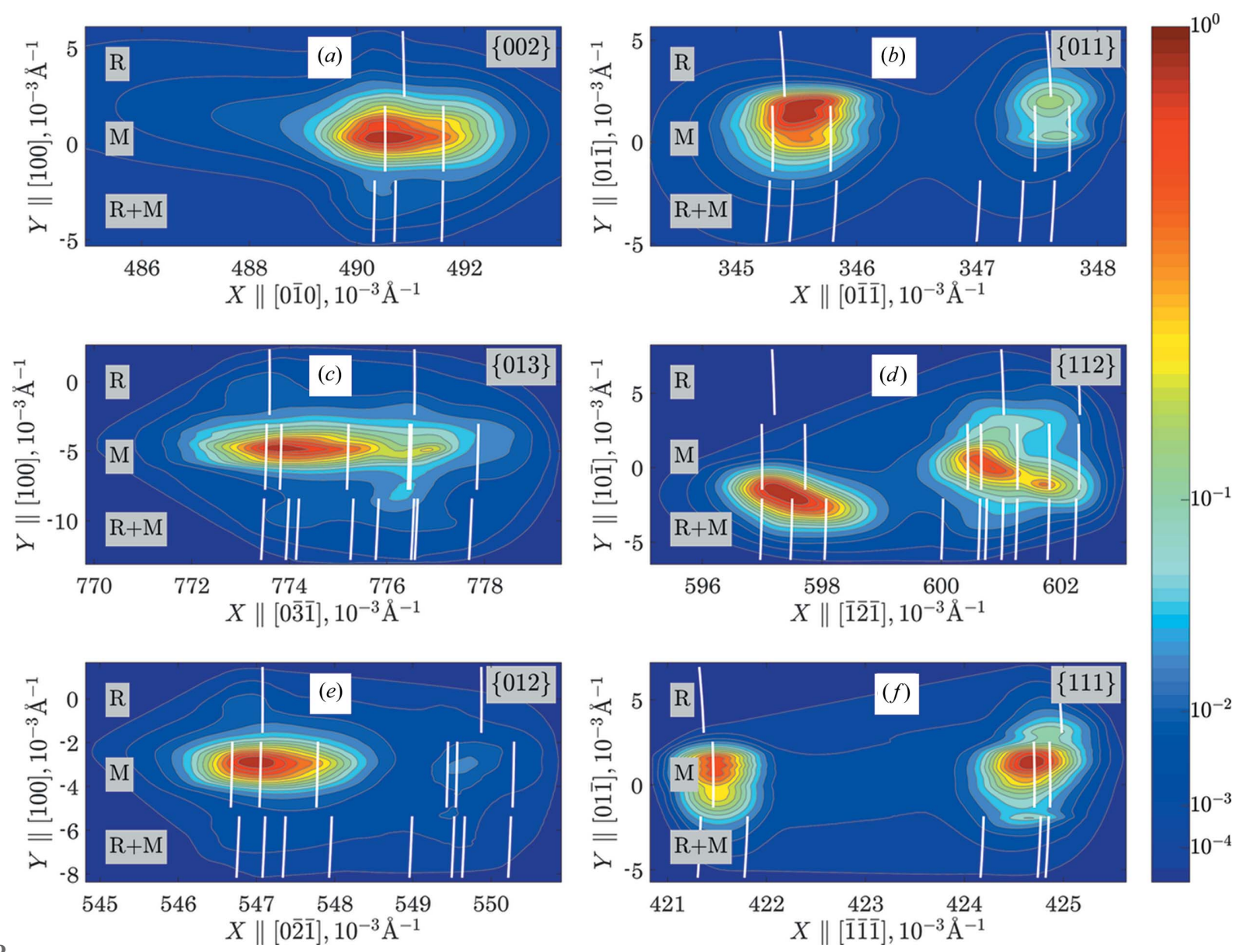

Figure 2

Reciprocal space maps of selected sets of reflections. (a) $\{002\} ;(b)\{011\} ;(c)\{013\} ;(d)\{112\} ;(e)\{012\} ;(f)\{111\}$. These intensity distributions are integrated along $\mathbf{Z}$ and drawn as functions of $X$ and $Y$. The horizontal axis, $X$, refers to the length of the scattering vector $(2 \sin \theta / \lambda)$, which is inversely proportional to the $d$ spacing. The radial separation between the peaks was used to infer the symmetry of a single domain. The vertical lines indicate the results of the fitting of the observed radial separations using different symmetries of the lattices. The best fit was achieved with a mixture of monoclinic and rhombohedral symmetry.

twinning of PZT45. This has also been discussed in previous X-ray diffraction (Gorfman et al., 2011) and polarized light microscopy (Bokov et al., 2010) studies of PZT and other ferroelectric single crystals (Gorfman \& Thomas, 2010; Datta et al., 2009). We converted these intensity distributions to a Cartesian $X Y Z$ coordinate system, where $\mathbf{X}$ is parallel to the scattering vector, while $\mathbf{Y}$ and $\mathbf{Z}$ are chosen to form a plane perpendicular to $\mathbf{X}$. The separation of Bragg peak components along the $X$ axis implies different lengths of the reciprocal lattice vectors, while any separations in the $Y Z$ plane imply different orientation of ferroelectric and/or ferroelastic domains. The $Y Z$ area, $\sigma_{Y Z}$, of the individual Bragg peak components ranges between $10^{-6}$ and $10^{-5} \AA^{-2}$, indicating estimated domain sizes in real space $\left[1 /\left(\sigma_{Y Z}\right)^{1 / 2}\right]$ ranging between thousands and hundreds of nanometres. A more precise evaluation of the domain sizes would require taking into account instrumental contributions, such as beam divergence, degree of X-ray beam monochromatization and detector pixel size.

Fig. 2 shows the $X Y$ reciprocal space maps (integrated along Z) of the six reflections $\{002\},\{011\},\{013\},\{122\},\{012\}$ and $\{111\}$. We analysed the separation of peaks along the $X$ axes in these and eight other reflections to infer the symmetry of a single domain following the procedure demonstrated previously (Gorfman \& Thomas, 2010; Datta et al., 2009; Gorfman et al., 2011; Vergentev et al., 2016). This procedure begins by marking the prominently sharp Bragg peaks in each map, determining their reciprocal space coordinates and building a list of unique peak-to-peak vectors within each map. It continues by choosing the crystal system and refining 'free' lattice parameters to the values that ensure the best possible match of measured and calculated separations of peaks along the direction of the $X$ axis. We tested the lattices of rhombohedral $(R)$, monoclinic $(M)$, and a combination of rhombohedral and monoclinic $(R+M)$ symmetries, in a manner similar to that previously reported (Gorfman et al., 2015). The $R$-phase lattice parameters were restricted as $a=b=c$ and $\alpha=$ $\beta=\gamma$. The monoclinic lattice parameters were restricted as $a=$ $b \neq c$ and $\alpha=\beta \neq \gamma .{ }^{3}$ The best fit was found for a coexistence of rhombohedral and monoclinic phases.

\footnotetext{
${ }^{3}$ These lattice parameters will look metrically monoclinic $\left(a_{\mathrm{m}} \neq b_{\mathrm{m}} \neq c_{\mathrm{m}}, \alpha_{\mathrm{m}}=\right.$ $\left.\gamma_{\mathrm{m}}=90^{\circ}, \beta_{\mathrm{m}} \neq 90^{\circ}\right)$ when the unit-cell vectors are transformed as $\mathbf{a}_{\mathrm{m}}=\mathbf{a}+\mathbf{b}$; $\mathbf{b}_{\mathrm{m}}=\mathbf{a}-\mathbf{b} ; \mathbf{c}_{\mathrm{m}}=\mathbf{c}$. In this case the mirror plane becomes parallel to $(010)_{\mathrm{m}}$.
} 
Several sets of high-resolution Bragg reflections were selected for the detailed analyses of the multi-scale responses of PZT45 to an electric field. Fig. 3(a) shows the $Y Z$ maps (integrated along $\mathbf{X}$ ) of the $\{002\}$ reflections, measured at four electric field states. The reflection has multiple sub-components, each presumably diffracted from a single set of ferroelectric/ferroelastic domains. We selected three such subcomponents as marked by the relevant $Y Z$ boxes in Fig. 3(a). Fig. 3(b) shows the $X$ dependence of the intensity distribution when intensity is integrated only in the marked $Y Z$ boxes and in the whole $Y Z$ plane. The $X$ positions of the COMs of these boxes are different, implying that the diffracting lattice planes have slightly different $d$ spacings. The response of each domain to the external electric field is also different, as shown in Figs. 3(c)-3(e). This may originate from different polarization directions with respect to the electric field and the scattering vectors. In the monoclinic $(\mathrm{Cm})$ domains (Vanderbilt \& Cohen, 2001), the polarization may lie in one of twelve $\{110\}$ planes. The external electric field was applied along one of the pseudocubic $\langle 001\rangle$ directions and thus could rotate the
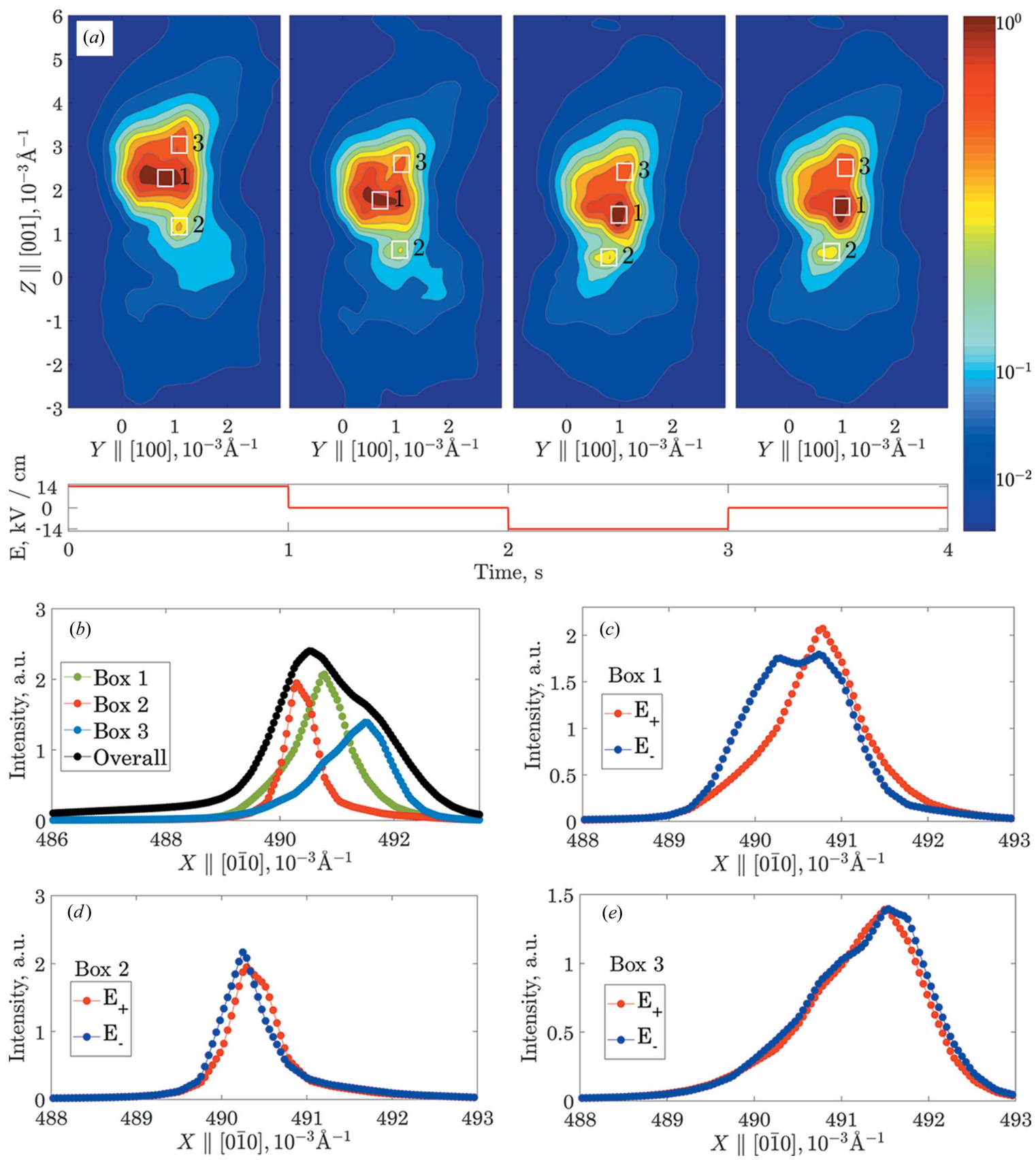

Figure 3

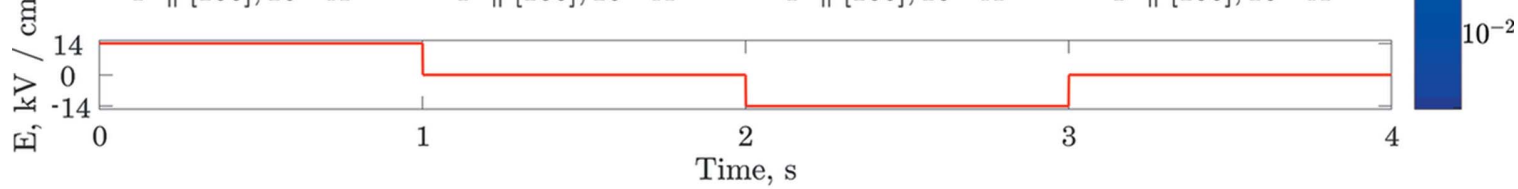

Response of the monoclinic domains with different orientations of polarization rotation planes with respect to the applied electric field. (a) $Y Z$

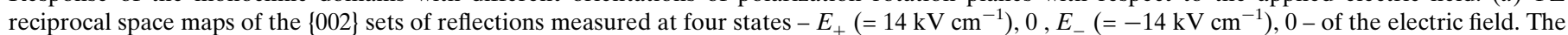
maps are integrated along $\mathbf{X}$. The $Y Z$ boxes marking the individual Bragg peak components are explicitly shown in the maps. $(b) X$ dependence of intensity distribution (integrated along $\mathbf{Y}$ and $\mathbf{Z}$ within the individually marked $Y Z$ boxes), showing that the diffracting planes of different domains have slightly different $d$ spacings. (c)-(e) Responses of three Bragg peak components to the switching of the electric field between +14 and $-14 \mathrm{kV}^{-1}$. 
polarization vector in each of the monoclinic domains. For those domains where the scattering vector appears to be out of the polarization rotation plane, the response might have a minor effect on the corresponding $d$ spacing (as in Boxes 2 and 3 ). On the other hand, those monoclinic domains where the scattering vector is on the polarization rotation plane should experience a stronger change in the $d$ spacing (e.g. Fig. $3 c$ ).

We now discuss how the number of domains included (specified by the number of marked peak components seen in the $Y Z$ plane) affects the average electric field induced strain. $\Delta X(E)$ is related to the effective piezoelectric coefficient, which describes the relative elongation/contraction of the crystal along the scattering vector in response to the applied electric field. Fig. 4( $a$ ) shows the $Y Z$ map and three boxes of different sizes (small, medium and large). Figs. $4(b)-4(d)$ show the corresponding $X$ scans for the small, the medium and the large $Y Z$ boxes under four states of the electric field. The small $Y Z$ box only selects a single domain and the corresponding shift of the $X$-scan profile describes an intrinsic response of the selected domain to an electric field. As the box size increases, it includes further peak components and its scan profile reflects the collective response from several domains. This accumulation, including mutual cancelling (when the strains in different domains have opposite signs), is shown in Fig. 4(e). Both the signs and the magnitudes of the COM shifts vary from domain to domain.

For the further discussion of our results, we should clarify that there are two relevant types of domains. One corresponds to ferroelastic domains, which are sometimes referred to as, for example, $90^{\circ}$ domains in tetragonal or $71 / 109^{\circ}$ domains in rhombohedral ferroelectrics. They are separated by straight domain walls and their sizes vary from hundreds of nano- metres to micrometres. The diffraction from these domains may be well separated in reciprocal space (e.g. 111 and $11 \overline{1}$ for rhombohedral ferroelectrics). Another type is the $180^{\circ}$ ferroelectric domains, for which there is no strain mismatch and which therefore cannot be separated by X-ray diffraction. However, if there is symmetry lowering (e.g. from rhombohedral to monoclinic) and polarization rotation in the crystal, these domains can have polarizations slightly rotated away from the higher-symmetry axes. We refer to them as $180^{\circ} \pm \varphi$ domains, where $\varphi$ stands for a small rotation angle. These domains are separated by the so-called $S$ walls (Fousek \& Janovec, 1969) embedded in the larger ferroelastic domains and can be observed using piezo-force scanning probe microscopy (Yao et al., 2011; Bai et al., 2004). Because of the small domain size and presumably smaller values of $\varphi$, the diffraction from these domains is usually harder to separate. However, in our high-resolution experiment, their different behaviours under electric fields make it possible to discriminate and analyse them. When $\varphi$ is not $0^{\circ}$, the monoclinic domains can contribute to the piezoelectric response through the rotation of the polarization direction.

We now discuss the response of the above domain types by analysing the $\{111\}$ Bragg peaks. These reflections contain two groups with different $X$ values (Fig. $2 f$ ) and so present the responses from both types of domains. Fig. 5(a) shows the $X$ profile, integrated within the full $Y Z$ range, covering all the sub-components of this peak. The parts of reflections with different values of $X$ were explicitly marked as Group 1 and Group 2. Figs. 5(b) and 5(c) show the $Y Z$ maps corresponding to each of the groups. We marked five boxes in Group 1 and three boxes in Group 2. The diffraction intensities were then integrated within these $Y Z$ boxes and plotted against the $X$
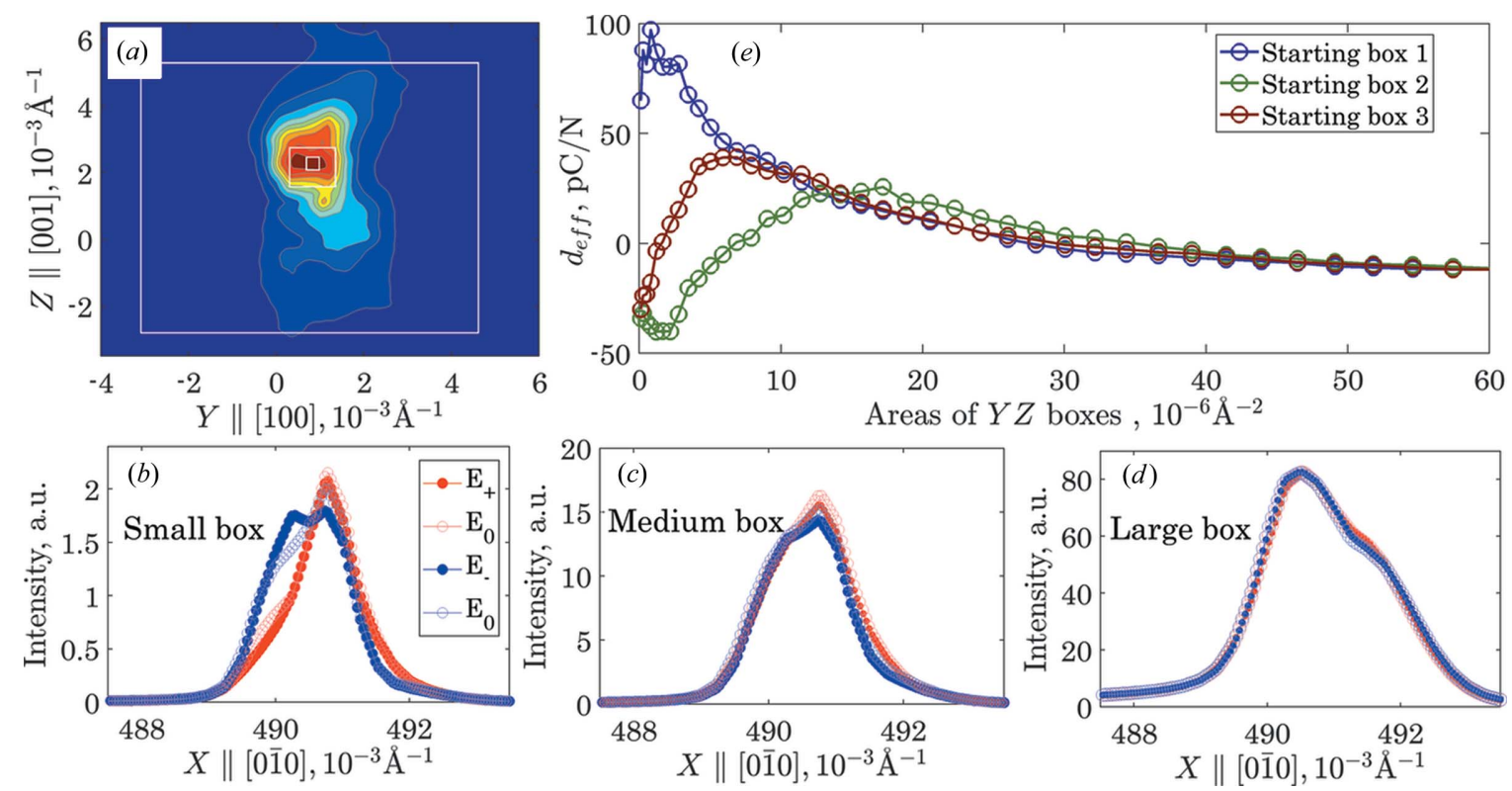

Figure 4

(a) Reciprocal space $(Y Z)$ map around the $\{002\}$ set of reflections with three different (small, medium and large) $Y Z$ boxes shown. (b)- $(d)$ The $X$ dependence of diffraction intensities, integrated within the small, medium and large boxes, for four different electric field states. (e) COM-changeinduced effective piezoelectric coefficients between the positive and zero fields for the three boxes defined in $(a)$ against the increasing box size in reciprocal space. 
values. Figs. 5(d) and 5(e) give examples of such plots for Box 2 in Group 1 and Group 2, respectively. They show peak shifts in $d$ spacing as well as intensity changes.

Fig. 6 shows how the electric field induced shift of the COM changes with increasing box size. We can decompose intrinsic and extrinsic contributions as follows: assuming the COM positions of each peak in Groups 1 and 2 are $X_{1}$ and $X_{2}$, and the areas under the curves are $I_{1}$ and $I_{2}$, we can write the position of the overall COMs as

$$
\langle X\rangle=\frac{I_{1} X_{1}+I_{2} X_{2}}{I_{1}+I_{2}} .
$$

The field-induced COM shift is

$$
\Delta\langle X\rangle=\frac{\left(\Delta I_{1} X_{1}+I_{1} \Delta X_{1}\right)+\left(\Delta I_{2} X_{2}+I_{2} \Delta X_{2}\right)}{I_{1}+I_{2}} .
$$

Assuming that $\Delta I_{1} \cong-\Delta I_{2}$ (the intensity change in each peak group is only due to the exchange between them), equation (2) can be rewritten as

$$
\Delta X=\frac{\Delta I_{1}\left(X_{1}-X_{2}\right)}{I_{1}+I_{2}}+\frac{I_{1} \Delta X_{1}+I_{2} \Delta X_{2}}{I_{1}+I_{2}} .
$$

The first part of equation (3) depends on the amount of intensity exchange $\left(\Delta I_{1}\right)$ and reflects the extrinsic effect due to the volume change of the domains via domain-wall motion. The second part depends on the individual COM shifts of Group $1\left(\Delta X_{1}\right)$ and Group $2\left(\Delta X_{2}\right)$. This part accumulates from several components constituting the groups. Each of the constituting domains (some of them are of $180^{\circ} \pm \varphi$ type) results from its own intrinsic electromechanical response (seen as a COM shift of the corresponding peak) and contributes to the cumulative strain (the COM shift of the entire group). The individual contributions are shown on either side of Fig. 6 with the smallest reciprocal space area, and the corresponding COM shifts are relatively high. These intrinsic COM shifts change with increasing $Y Z$ box size until they reach the 'accumulated intrinsic' values. Notably, the size of the box above which the COM shift saturates is very similar to that of the $\{002\}$ peaks (Fig. 4e). The COM shift for the entire Group 1 and Group 2 comes from the sum of all the constituent peaks. Finally, the extrinsic and accumulated intrinsic contributions are calculated according to equation (3). The overall effective local piezoelectric coefficients are obtained from the relative COM shifts and plotted in the middle of Fig. 6. These values show that the extrinsic contribution is much larger than the accumulated intrinsic contribution: for this particular crystal and crystallographic direction, the extrinsic contribution amounts to over $80 \%$ of the total response.

Overall, our analyses on multiple Bragg reflections show that there are several contributions to the enhancement of the electromechanical response in PZT45 crystals. First, the freedom of the polarization to rotate within the monoclinic mirror plane enhances the pure intrinsic effect. Second, the $180^{\circ} \pm \varphi$ domains may cause the cumulative piezoelectric response to decrease, because the individual contributions may have different signs and cancel each other. Since the materials used in industrial devices are usually poled, the cancelling effects are reduced by converting $180^{\circ} \pm \varphi$ into $\pm \varphi$

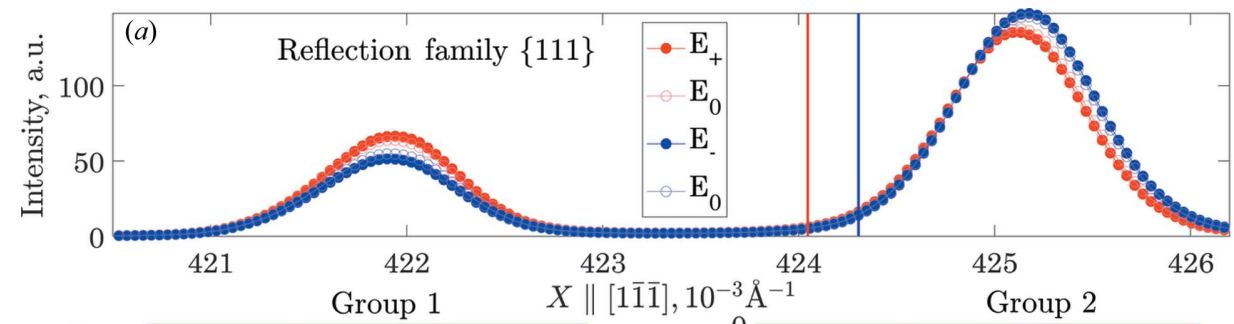

Figure 5
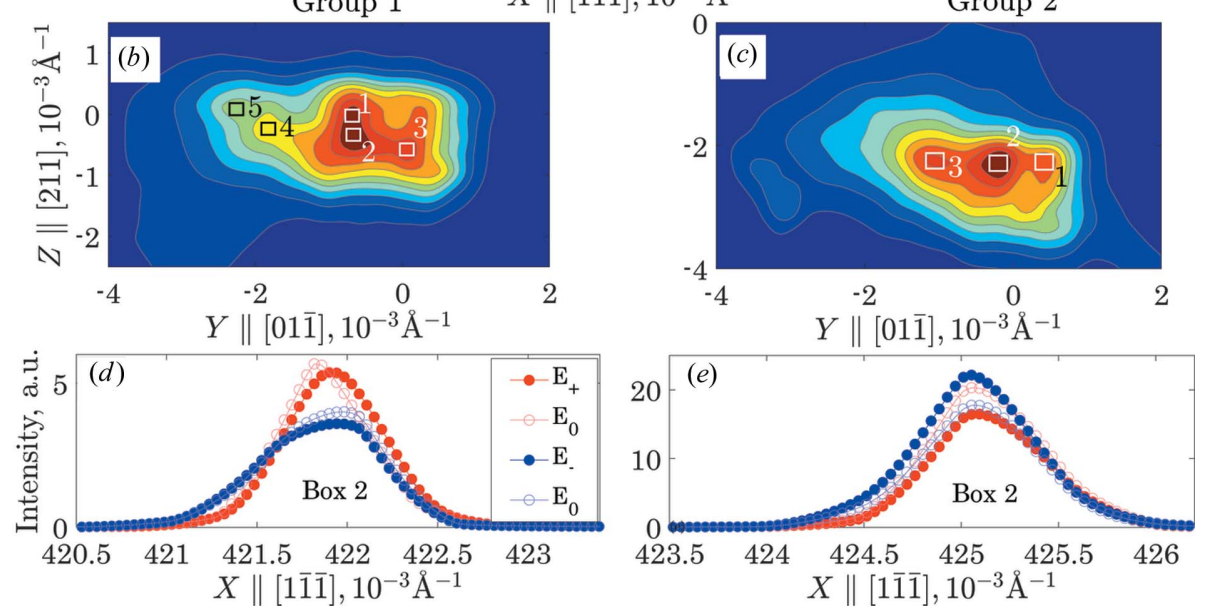

(a) The $X$ dependence of the diffraction intensity around $\{111\}$ reflections, integrated within the full $Y Z$ range. The vertical red and blue lines mark the COM positions corresponding to the $E_{+}$and $E_{-}$states. $(b)-(c) Y Z$ dependence of the diffraction intensity integrated within two ranges of $X$, corresponding to Group 1 and Group 2 in panel $(a)$. Several boxes are marked to show the positions of Bragg peak sub-components. $(d)-(e)$ Integrated intensities within one $Y Z$ box against $X$ under four states of field. $(d)$ corresponds to Box 2 in Group 1 and (e) to Box 2 in Group 2. 


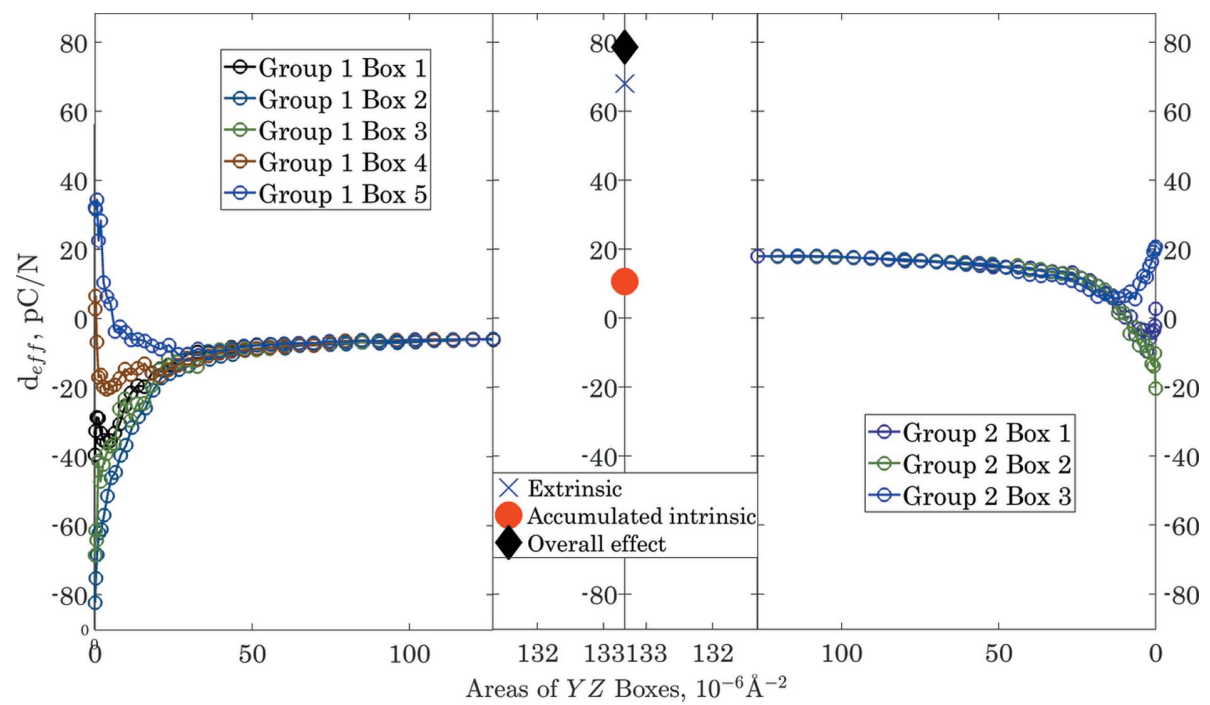

Figure 6

The effective local piezoelectric coefficients, calculated from the COM shift between positive $\left(E_{+}\right)$and zero $(0)$ electric fields with increasing $Y Z$ box sizes. The left part corresponds to the five boxes in Group 1 and the right part to the three boxes in Group 2; the boxes and the groups are explicitly defined in Fig. 5. The middle part shows the contributions to the effective piezoelectric contributions from the extrinsic and accumulated intrinsic effects.

domains without altering the density of the $\mathrm{S}$ walls. Third, the extrinsic effect can be large where the classic ferroelastic (non$180^{\circ} \pm \varphi$ ) domains are present and where active volume exchange between different strain domains is possible.

\section{Conclusions}

In conclusion, with the investigation of this PZT45 crystal, we demonstrate the application of a new in situ high-resolution diffraction experiment and analysis to investigate the multiscale piezoelectric activities in multi-domain ferroelectric/ ferroelastic single crystals. We have shown, in particular, how the separation of individual Bragg peak components and the changes of their positions/intensities under an external electric field can help separate intrinsic and extrinsic contributions to the piezoelectric effect. Such an approach can also be generalized for many other external perturbations and augmented with modulation (Chernyshov et al., 2011) or principal component analysis (Caliandro et al., 2015). The suggested experimental methods exploiting the development of fast pixel detectors and a data analysis framework have a high potential for obtaining insights into complex systems to understand the origin of crystal physical properties on different length and time scales.

\section{Funding information}

NZ acknowledges support by the National Natural Science Foundation of China (grant No. 61604123) and Fundamental Research Funds for the Central Universities of China. ZGE and BW acknowledge support by the United States Office of Naval Research (ONR grants No. N00014-12-1-1045 and No. N00014-16-13106) and the Natural Sciences and Engineering Research Council of Canada (NSERC, grant No. 203773). SG and $\mathrm{HC}$ acknowledge the support of Bundesministerium für Bildung und Forschung (award No. 05K13PSA).

\section{References}

Bai, F., Li, J. \& Viehland, D. (2004). Appl. Phys. Lett. 85, A. A., Long, X. \& Ye, Z.-G. (2010). Phys. Rev. B, 81, 172103.

Bokov, A. A., Long, X. \& Ye, Z.-G. (2010). Phys. Rev. B, 81, 172103. Caliandro, R., Guccione, P., Nico, G., Tutuncu, G. \& Hanson, J. C. (2015). J. Appl. Cryst. 48, 1679-1691.

Chernyshov, D., van Beek, W., Emerich, H., Milanesio, M., Urakawa, A., Viterbo, D., Palin, L. \& Caliandro, R. (2011). Acta Cryst. A67, 327-335.

Choe, H., Heidbrink, S., Ziolkowski, M., Pietsch, U., Dyadkin, V., Gorfman, S. \& Chernyshov, D. (2017). J. Appl. Cryst. 50, 975977.

Daniels, J. E., Cozzan, C., Ukritnukun, S., Tutuncu, G., Andrieux, J., Glaum, J., Dosch, C., Jo, W. \& Jones, J. L. (2014). J. Appl. Phys. 115, 224104.

Datta, K., Gorfman, S. \& Thomas, P. A. (2009). Appl. Phys. Lett. 95, 251901.

Devonshire, A. F. (1954). Adv. Phys. 3, 85-130.

Durbin, M. K., Jacobs, E. W., Hicks, J. C. \& Park, S.-E. (1999). Appl. Phys. Lett. 74, 2848-2850.

Dyadkin, V., Pattison, P., Dmitriev, V. \& Chernyshov, D. (2016). J. Synchrotron Rad. 23, 825-829.

Fousek, J. \& Janovec, V. (1969). J. Appl. Phys. 40, 135-142.

Gorfman, S., Keeble, D. S., Bombardi, A. \& Thomas, P. A. (2015). J. Appl. Cryst. 48, 1543-1550.

Gorfman, S., Keeble, D. S., Glazer, A. M., Long, X., Xie, Y., Ye, Z.-G., Collins, S. \& Thomas, P. A. (2011). Phys. Rev. B, 84, 020102.

Gorfman, S. \& Thomas, P. A. (2010). J. Appl. Cryst. 43, 1409-1414.

Haertling, G. H. (1999). J. Am. Ceram. Soc. 82, 797-818.

Hall, D. A., Mori, T., Withers, P. J., Kungl, H., Hoffmann, M. J. \& Wright, J. (2008). Mater. Sci. Technol. 24, 927-933.

Hall, D. A., Steuwer, A., Cherdhirunkorn, B., Mori, T. \& Withers, P. J. (2004). J. Appl. Phys. 96, 4245-4252.

Helke, G., Lubitz, K., Heywang, W., Lubitz, K. \& Wersing, W. (2008). Piezoelectricity, edited by W. Heywang, K. Lubitz \& W. Wersing, pp. 89-130. Berlin, Heidelberg: Springer-Verlag.

Howard, C. J. \& Stokes, H. T. (2005). Acta Cryst. A61, 93-111. 
Jones, J. L. (2007). J. Electroceram. 19, 67-79.

Jones, J. L., Hoffman, M., Daniels, J. E. \& Studer, A. J. (2006). Appl. Phys. Lett. 89, 092901.

Jones, J. L., Slamovich, E. B. \& Bowman, K. J. (2005). J. Appl. Phys. 97, 034113.

Kugel, V. D. \& Cross, L. E. (1998). J. Appl. Phys. 84, 2815-2830.

Mitchell, R. H. (2002). Perovskites: Modern and Ancient. Thunder Bay: Almaz Press.

Oddershede, J., Majkut, M., Cao, Q., Schmidt, S., Wright, J. P., Kenesei, P. \& Daniels, J. E. (2015). J. Appl. Cryst. 48, 882-889.

Torgashev, V. I., Shirokov, V. B., Prokhorov, A. S. \& Shuvalov, L. A. (2005). Crystallogr. Rep. 50, 637-645.

Uchino, K. (2015). Sci. Technol. Adv. Mater. 16, 046001.

Vanderbilt, D. \& Cohen, M. H. (2001). Phys. Rev. B, 63, 094108.
Vergentev, T., Bronwald, I., Chernyshov, D., Gorfman, S., Ryding, S. H. M., Thompson, P. \& Cernik, R. J. (2016). J. Appl. Cryst. 49, 1501-1507.

Vergentev, T. Y., Dyadkin, V. \& Chernyshov, D. Y. (2015). J. Surf. Investig. X-ray Synchrotron Neutron Tech. 9, 436-441.

Wersing, W., Heywang, W., Beige, H. \& Thomann, H. (2008). Piezoelectricity, edited by W. Heywang, K. Lubitz \& W. Wersing, pp. 37-87. Berlin, Heidelberg: Springer-Verlag.

Xie, Y. (2013). PhD thesis, Simon Fraser University, Burnaby, Canada.

Yao, J., Yan, L., Ge, W., Luo, L., Li, J., Viehland, D., Zhang, Q. \& Luo, H. (2011). Phys. Rev. B, 83, 054107.

Zhang, N., Yokota, H., Glazer, A. M., Keen, D. A., Gorfman, S., Thomas, P. A., Ren, W. \& Ye, Z.-G. (2018). IUCrJ, 5, 73-81. 